\title{
Cholesterol-Binding by the Yeast CAP Family Member Pry1 Requires the Presence of an Aliphatic Side Chain on Cholesterol
}

Rabih Darwiche and Roger Schneiter

University of Fribourg, Department of Biology, 1700 Fribourg, Switzerland

"Corresponding author: Schneiter R, University of Fribourg, Department of Biology, Chemin du Musée 10, 1700 Fribourg, Switzerland, E-mail: roger.schneiter@unifr.ch

Receiving date: Mar 01, 2016; Accepted date: May 02, 2016; Publishing date: May 07, 2016

Copyright: @ 2016 Darwiche R, et al. This is an open-access article distributed under the terms of the Creative Commons Attribution License, which permits unrestricted use, distribution, and reproduction in any medium, provided the original author and source are credited.

\begin{abstract}
Pathogen-related yeast protein 1 (Pry1) is a Saccharomyces cerevisiae member of the CAP/SCP/TAPS superfamily. Although, CAP proteins have been proposed to be implicated in a number of physiological processes, such as pathogen virulence, sperm maturation and fertilization, host-pathogen interactions and defense mechanisms, the molecular mode of action of these proteins is poorly understood. CAP proteins are mostly secreted and they are stable in the extracellular space over a wide a range of conditions. All members of this superfamily contain a common CAP domain of approximately 150 amino acids, which adopts a unique $\alpha-\beta-\alpha$ sandwich fold. We have previously shown that the yeast CAP family members act as sterol-binding and -export proteins in vivo and that the Pry proteins bind cholesterol and cholesteryl acetate in vitro. The conserved CAP domain of Pry1 is necessary and sufficient for sterol binding. Based on these observations, it is conceivable that CAP proteins exert their biological function through a common mechanism, such as binding and sequestration of sterols or related small hydrophobic compounds. Here we analyze the ligand specificity of Pry1 in more detail and show that the presence of the aliphatic isooctane side chain of the sterol but not the 3-hydroxyl group is important for binding to Pry1.
\end{abstract}

Keywords: Pathogen-related yeast 1 (Pry1); CAP/SCP/TAPS superfamily; Sterols; Steroids; In vitro Ligand-binding assay; Saccharomyces cerevisiae

\section{Introduction}

The CAP/SCP/TAPS superfamily of proteins (cysteine-rich secretory proteins, antigen 5 (Ag5), pathogenesis related 1 proteins (PR-1)/sperm coating proteins/Tpx-1/Ag5/PR-1/Sc7; Pfam accession number PF00188) comprises more than 4500 members in over 1500 species and family members are found in all kingdoms of life. CAP proteins have been implicated in a wide variety of processes, including immune defense in mammals and plants, pathogen virulence, sperm maturation and fertilization, venom toxicity, and prostate and brain cancers. CAP proteins are mostly secreted and all members of this superfamily share a common CAP domain of approximately 150 amino acids, which adopts a unique $\alpha-\beta-\alpha$ sandwich fold and is connected by flexible loop regions. The overall structural conservation within the CAP protein family suggests that these proteins exert fundamentally similar functions. However, the molecular mode of action of this protein family has remained enigmatic [1-3].

The genome of the yeast Saccharomyces cerevisiae encodes for three CAP family members, two of which, Pryl and Pry2, are secreted, whereas Pry3 is a cell wall-associated protein. Pry1 and Pry2 share a redundant function in the export of acetylated cholesterol and cells lacking both $P R Y 1$ and $P R Y 2$ have a complete block in secretion of the acetylated lipid in vivo [4]. Purified Pry1 and Pry2 bind both free cholesterol and cholesteryl acetate in vitro [4]. The sterol binding and export function maps to the CAP domain, as its expression alone is efficient to rescue sterol export in cells lacking Pry proteins [4]. Additionally, expression of human CAP member, CRISP2 (cysteinerich secretory protein 2), or the Schistosoma mansoni venom allergen like protein 4, SmVAL4, in yeast rescues the sterol export defect of a pry1 1 pry $2 \Delta$ double mutant and purified CRISP2 or SmVAL4 proteins bind cholesterol in vitro, indicating that cholesterol binding and export is a conserved function of diverse CAP superfamily members [4-5]. Computational modeling indicates that ligand binding could occur through displacement of a flexible loop, termed the caveolin-binding motif (CBM) that is rich in aromatic side chains [6]. Point mutations within this motif abrogate sterol export and binding while mutations of residues located outside the CBM had no effect on lipid export and binding. The CBM thus appears to play a key role in the ability of CAP proteins to bind cholesterol [5-7].

\section{Results and Discussion}

Cells lacking $P R Y 1$ and $P R Y 2$ are hypersensitive to the plant oil eugenol and this hypersensitivity is rescued by expression of human CRISP2 [3]. To examine whether Pryl would directly bind the plant oil eugenol (2-methoxy-4-(2-propenyl) phenol), a member of the allylbenzene class of compounds that is present in clove oil, nutmeg, cinnamon, and bay leaf, and is used as local antiseptic and anesthetic [8-12], we performed a competition-binding assay with purified Pry1 and $[3 \mathrm{H}]$-cholesterol. Addition of an equimolar amount $(50 \mathrm{pmol})$ of unlabeled eugenol to the labeled cholesterol resulted in a reduction in binding of the radiolabel cholesterol to Pryl. Addition of an excess of eugenol (100-5000 pmol) reduced binding of the radiolabeled cholesterol even further (Figure 1). These experiments reveal that eugenol efficiently competes with [3H]-cholesterol for binding to Pry1 protein, indicating that both cholesterol and eugenol compete for the same or an overlapping binding site. Thus, Pryl not only binds free sterols but also small hydrophobic compounds and may thereby protect cellular membranes from a potential detrimental action of eugenol and related small membrane perturbing agents. 
Thus, while sterols and eugenol are ligands that bind CAP family members, there may be other ligands that are potentially more relevant under particular physiological conditions. As part of an ongoing effort to identify endogenous ligand(s) of Pryl, we probe binding of small hydrophobic compounds in vitro using a competition binding assay [9-10].

Therefore, the ability of a variety of unlabeled sterols and steroids to compete with radiolabeled cholesterol for binding to Pry1 was measured [11-12]. Natural phytosterols like stigmasterol and sitosterol and the fungal sterol ergosterol have a chemical structure that is very similar to cholesterol and all three of these sterols competed efficiently with cholesterol for binding to Pry1 (Figure 2). Similarly, epicholesterol, a structural isomer of cholesterol with a $3 \alpha$, instead of the normal 3b-hydroxyl group, competed efficiently for binding to Pry1. Similarly, precursors in cholesterol biosynthesis, such as lanosterol and desmosterol competed efficiently for binding to Pry1. On the other hand, the cholesterol synthesis inhibitor U18666A failed to compete (Figure 2).

Further analysis of the in vitro substrate specificity of Pryl indicated that steroids such as pregnenolone, progesterone, and androstenol, which all lack the aliphatic side chain that is present in cholesterol, failed to compete with radiolabeled cholesterol for binding to Pry1 (Figure 3). However, (+)-4-cholesten-3-one, 7-ketocholesterol and epoxycholesterol, which harbor modifications in the ring system, but contain an isooctane side chain, competed for binding to Pry1 (Figure 3). Thus, the presence of the isooctane side chain is crucial for sterol binding to Pryl.

Taken together, our results show that Pryl is a cholesterol-binding protein that binds natural sterols, sterol precursors and small hydrophobic ligands such as eugenol. The structure of the competing sterols and that of the non-competing steroids further indicates that sterol binding to Pryl requires the presence of an aliphatic side chain. Different substitutions in the tetracyclic ring structure, particularly in the $\mathrm{A}$ and $\mathrm{B}$ ring, or modifications on the 3-hydroxyl group, however, do not affect binding to the protein. These results open the possibility that small compounds such as isooctane may be sufficient to block binding of sterols to Pryl and possibly other CAP superfamily members. Isooctane may thus be sufficient to neutralize the action of CAP proteins under different physiological settings, including venom toxicity or pathogen virulence.

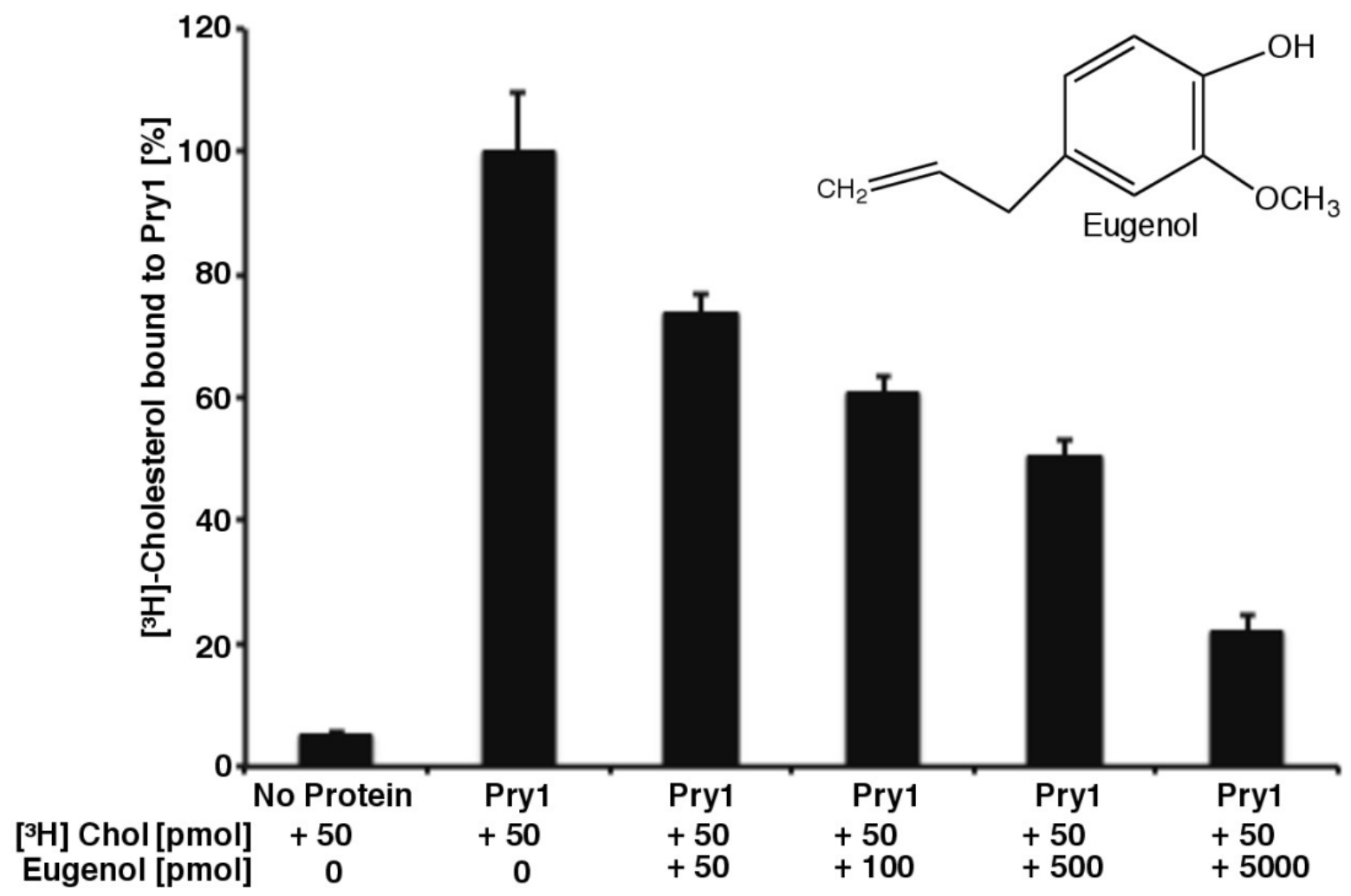

Figure 1: Pryl protein binds eugenol: Purified Pryl protein $(100 \mathrm{pmol})$ was incubated with [3H]-cholesterol $(50$ pmol $)$ as a ligand in the absence $(0 \mathrm{pmol})$, equal amount $(50 \mathrm{pmol})$, or increasing amounts $(100-5000 \mathrm{pmol})$ of eugenol and binding of the radioligand to the protein was measured. The $100 \%$ value refers to maximum Pryl binding capacity in the absence of unlabeled ligand. Values are means \pm standard deviations of three independent experiments. 


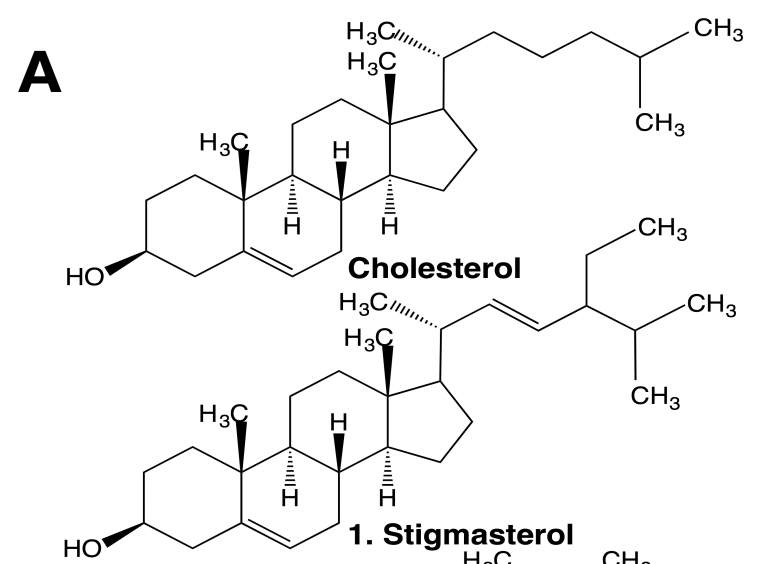

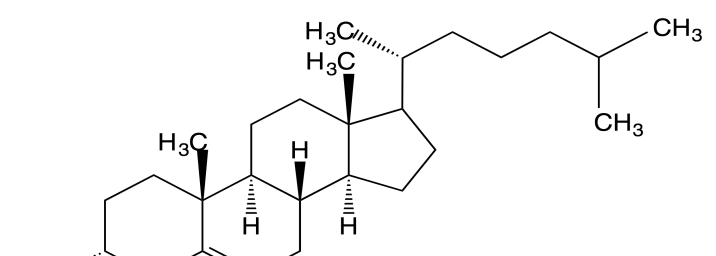

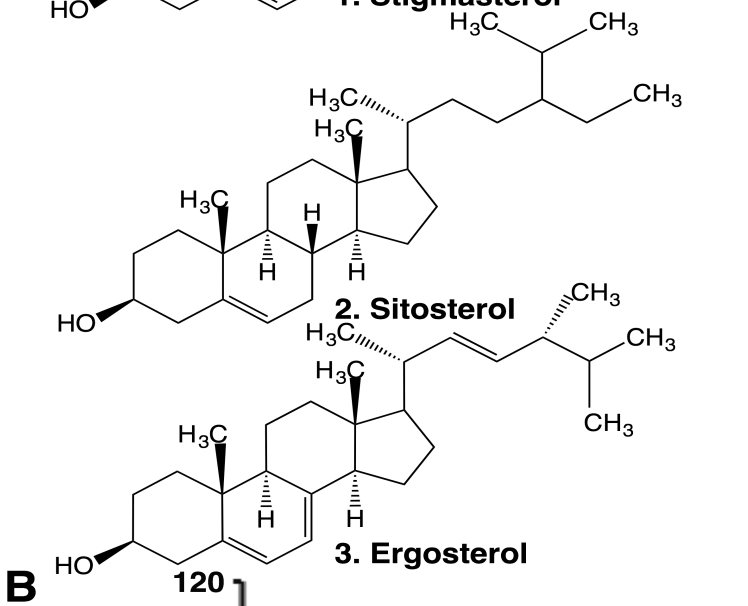

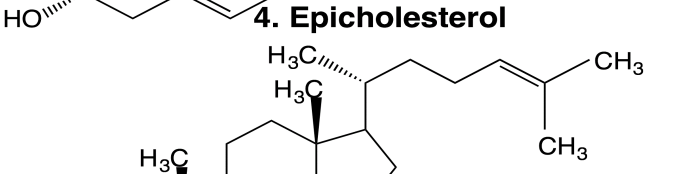

B

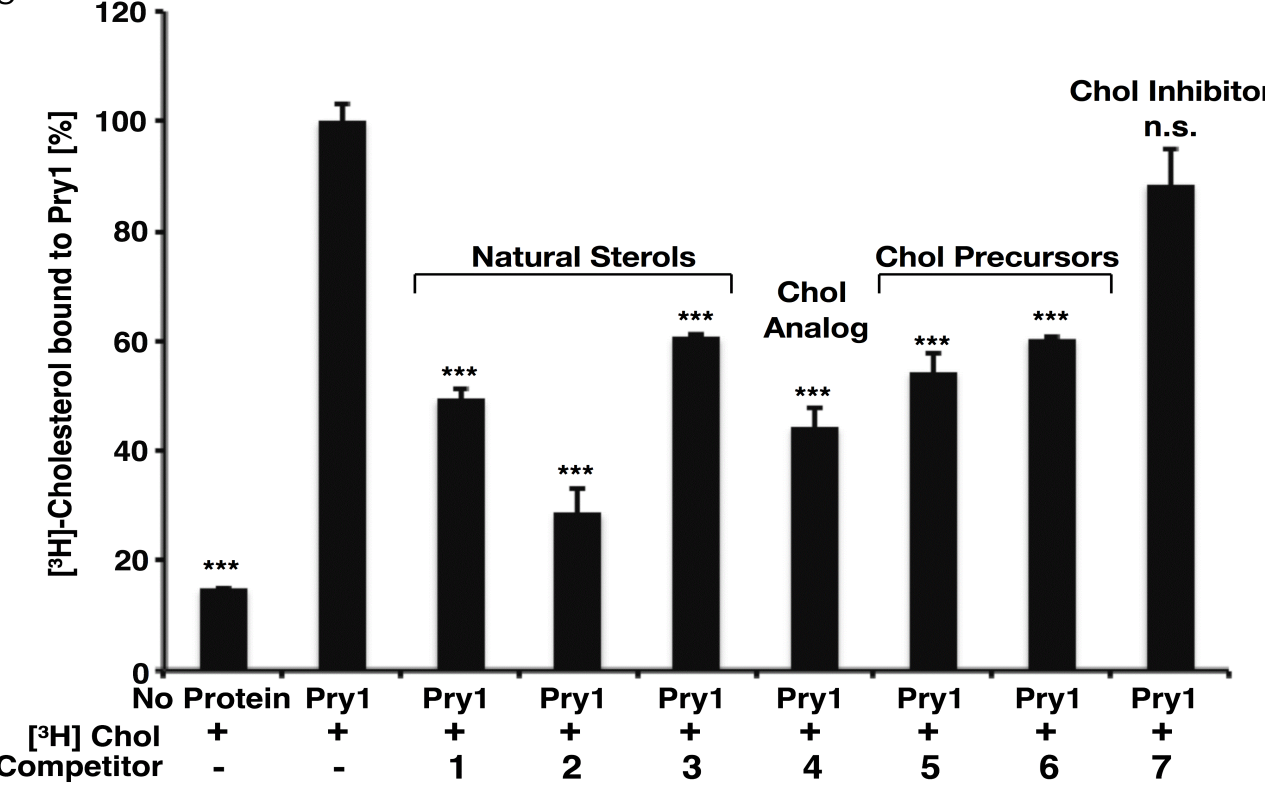

Figure 2: Specificity of sterol binding by Pry1. A) Structures of cholesterol and of the unlabeled sterols tested for their ability to compete with $[3 \mathrm{H}]$-cholesterol for binding to Pryl. B) Competitive binding of radiolabeled cholesterol to Pryl by unlabeled sterol precursors and analogues. Each binding reaction contained $50 \mathrm{pmol}$ of the indicated sterol and an equal amount of [3H]-cholesterol. Values are means \pm standard deviations of three independent experiments. Asterisks denote statistical significance ( ${ }^{* \star *} \mathrm{P}<0.0001$; n.s. (non-significant)). 

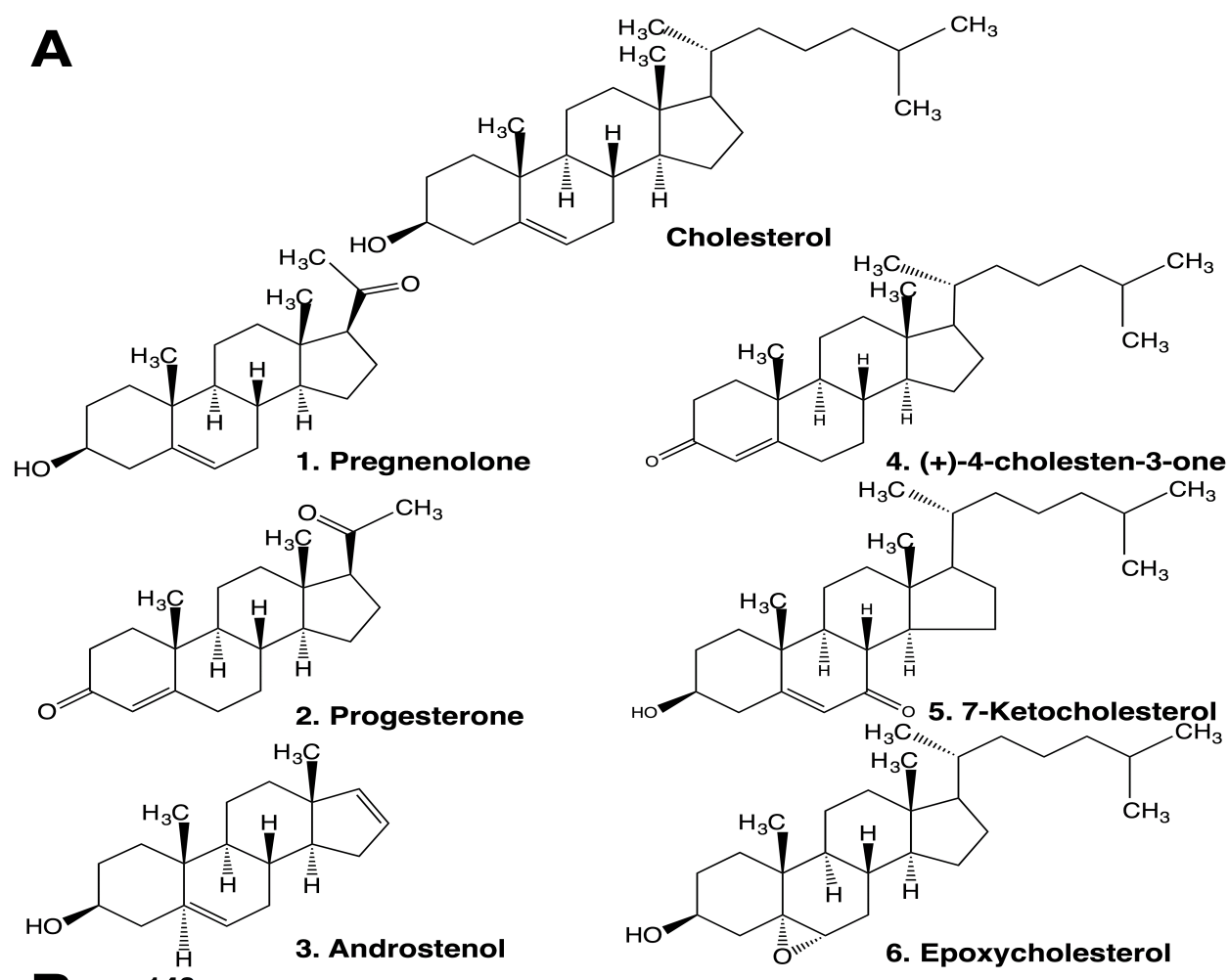

B

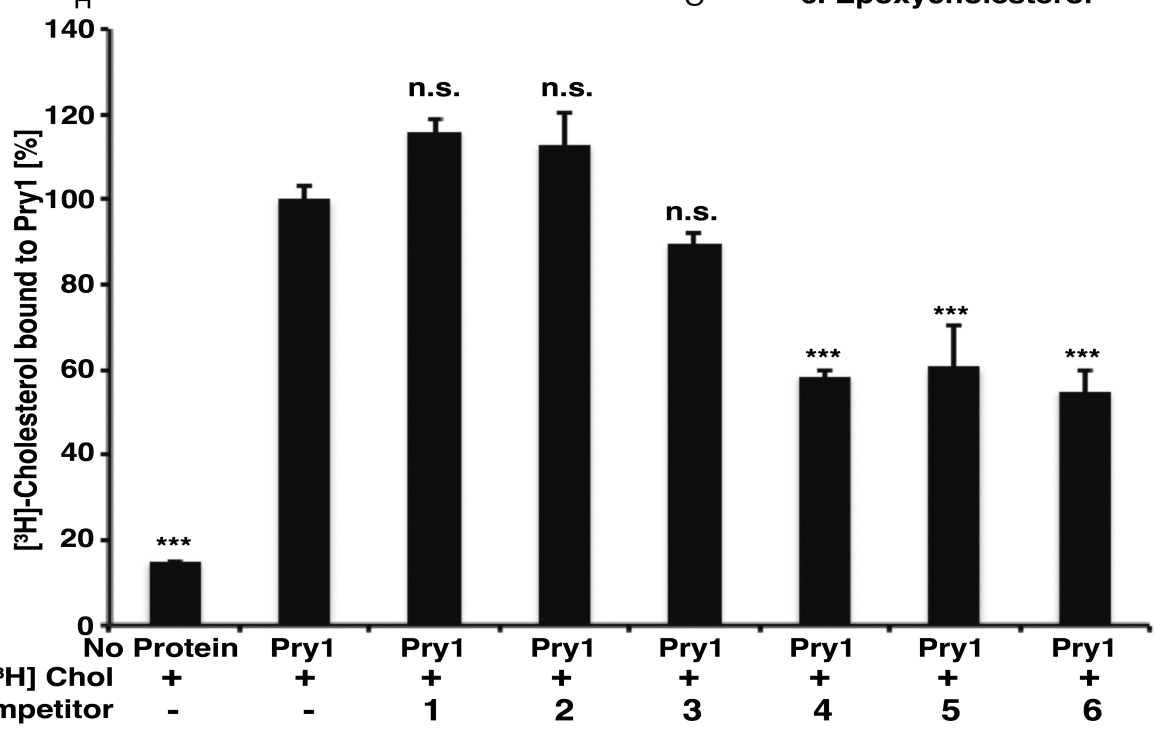

Figure 3: Ligand specificity for Pry1. A) Structures of cholesterol and of unlabeled steroids and sterols tested for their ability to compete with $[3 \mathrm{H}]$-cholesterol for binding to Pry1. B) Competitive binding of the indicated ligands. Each reaction contained 50 pmol of the indicated steroid and an equal amount of $[3 \mathrm{H}]$-cholesterol. Values are means \pm standard deviations of three independent experiments. Asterisks denote statistical significance $\left({ }^{* * *} \mathrm{P}<0.0001 ;\right.$ n.s. (non-significant)). 
Citation: Darwiche R, Schneiter R (2016) Cholesterol-Binding by the Yeast CAP Family Member Pry1 Requires the Presence of an Aliphatic Side Chain on Cholesterol. J Steroids Hormon Sci 7: 172. doi:10.4172/2157-7536.1000172

Page 5 of 5

\section{Acknowledgements}

We thank Stéphanie Cottier for critically reading the manuscript. This work was supported by the Swiss National Science Foundation.

\section{References}

1. Schneiter R, Di-Pietro A (2013) The CAP protein superfamily: function in sterol export and fungal virulence. Biomol Concepts 4: 519-525.

2. Gibbs GM, Roelants K, O'Bryan MK (2008) The CAP superfamily: cysteine-rich secretory proteins, antigen 5 , and pathogenesis-related proteins--roles in reproduction, cancer, and immune defense. Endocr Rev 29:865-897.

3. Cantacessi C, Campbell BE, Visser A, Geldhof P, Nolan MJ, et al. (2009) A portrait of the "SCP/TAPS" proteins of eukaryotes--developing a framework for fundamental research and biotechnological outcomes. Biotechnol Adv 27: 376-388.

4. Choudhary V, Schneiter R (2012) Pathogen-related yeast (PRY) proteins and members of the CAP superfamily are secreted sterol-binding proteins. Proc Natl Acad Sci U S A 109: 16882-16887.

5. Kelleher A, Darwiche R, Rezende WC, Farias LP, Leite LC, et al. (2014) Schistosoma mansoni venom allergen-like protein 4 (SmVAL4) is a nove lipid-binding SCP/TAPS protein that lacks the prototypical CAP motifs. Acta Crystallogr D Biol Crystallogr 70: 2186-2196.
6. Eberle HB, Serrano RL, Fullekrug J, Schlosser A, Lehnmann WD, et al. (2002) Identification and characterization of a novel human plant pathogenesis-related protein that localizes to lipid-enriched microdomains in the Golgi complex. J Cell Sci 115: 827-838.

7. Choudhary V, Darwiche R, Gfeller D, Zoete V, Michielin O, et al. (2014) The caveolin-binding motif of the pathogen-related yeast protein Pry1, a member of the CAP protein superfamily, is required for in vivo export of cholesteryl acetate. J Lipid Res 55: 883-894.

8. Bakkali F, Averbeck S, Averbeck D, Idaomar M (2008) Biological effects of essential oils--a review. Food Chem Toxicol 46: 446-475.

9. Infante RE, Radhakrishnan A, Abi-Mosleh L, Kinch LN, Wang ML (2008) Purified NPC1 protein: II. Localization of sterol binding to a 240 -amino acid soluble luminal loop. J Biol Chem 283: 1064-1075.

10. Im YJ, Raychaudhuri S, Prinz WA, Hurley JH (2005) Structural mechanism for sterol sensing and transport by OSBP-related proteins. Nature 437: 154-158.

11. Jacquier N, Schneiter R (2012) Mechanisms of sterol uptake and transport in yeast. J Steroid Biochem Mol Biol 129: 70-78.

12. Tiwari R, Köffel R, Schneiter R (2007) An acetylation/deacetylation cycle controls the export of sterols and steroids from S. cerevisiae. EMBO J 26: 5109-5119. 